Pacific

Journal of

Mathematics

ON ROUGH-ISOMETRY CLASSES OF HILBERT GEOMETRIES

THOMAS FOERTSCH

Volume $245 \quad$ No. 2

April 2010 


\title{
ON ROUGH-ISOMETRY CLASSES OF HILBERT GEOMETRIES
}

\author{
THOMAS FOERTSCH
}

\begin{abstract}
We prove that Hilbert geometries on uniformly convex Euclidean domains with $\boldsymbol{C}^{\mathbf{2}}$-boundaries are roughly isometric to the real hyperbolic spaces of corresponding dimension.
\end{abstract}

\section{Introduction}

Hilbert geometries generalize the Klein model of the real hyperbolic space from ellipsoids in $\mathbb{E}^{n}$, the $n$-dimensional Euclidean space, to arbitrary bounded convex subsets of $\mathbb{E}^{n}$. Karlsson and Noskov [2002] provide necessary conditions as well as sufficient conditions on the boundary of such a convex subset in order for its associated Hilbert geometry to be Gromov hyperbolic. Benoist [2003] even precisely determined such convex subsets, the associated Hilbert geometries of which are Gromov hyperbolic. Namely, such a bounded convex subset yields a Gromov hyperbolic Hilbert geometry if and only if its Euclidean boundary is locally the graph of a "quasisymmetrically convex" function.

Benoist [2006] proved that every two-dimensional Gromov hyperbolic Hilbert geometry is quasi-isometric to the real hyperbolic space of corresponding dimension. Here he also provides examples of Hilbert geometries in dimension $\geq 3$ which are not quasi-isometric to real hyperbolic spaces.

For related discussions of non-Gromov hyperbolic Hilbert geometries, see also [Bernig 2009; Bletz-Siebert and Foertsch 2007; Colbois and Verovic 2008; Colbois et al. 2008].

Restricting their attention to so-called strictly (or, as one might prefer, uniformly) convex domains, Colbois and Verovic [2004] proved that the Hilbert geometries of such domains are bi-Lipschitz equivalent to the real hyperbolic space of corresponding dimension.

The purpose of this paper is to prove that such Hilbert geometries are even rough-isometric to the real hyperbolic spaces of corresponding dimension.

MSC2000: primary 53C60; secondary 51F99.

Keywords: Hilbert geometries, Gromov hyperbolicity, rough isometry. 
Recall that a map $f: X \longrightarrow Y$ between metric spaces is called a rough-isometric embedding if there exists some $k \geq 0$ such that

$$
\left|x x^{\prime}\right|-k \leq\left|f(x) f\left(x^{\prime}\right)\right| \leq\left|x x^{\prime}\right|+k \text { for all } x, x^{\prime} \in X .
$$

If, moreover, for all $y \in Y$ there exists an $x \in X$ such that $|y f(x)| \leq k$, then $f$ is called a rough isometry.

Recall further that Gromov hyperbolicity is a rough-isometry invariant, and in the course setting of Gromov hyperbolic spaces, what one is generally interested in are the corresponding rough-isometry classes.

Theorem 1.1. Let $D$ be an open, bounded convex domain in $\mathbb{E}^{n}$. Suppose further that the boundary $\partial D$ is of class $C^{2}$ and the curvature of $\partial D$ is nonzero everywhere. Then the Hilbert geometry $\left(D, h_{\kappa}^{D}\right)$ associated with $D$ is rough-isometric to $\mathbb{H}_{\kappa}^{n}$.

The proof relies on the equivalence of rough-isometry classes of visual, Gromov hyperbolic spaces and bi-Lipschitz classes of their boundaries at infinity. We recall in Section 2 the precise definition of Hilbert geometries and summarize such facts on Gromov hyperbolic spaces as will be needed in the proof of Theorem 1.1. In Section 3 we give proofs of some elementary geometric lemmata, which will also be quoted in the proof of Theorem 1.1 in Section 4.

\section{Preliminaries}

2.1. Hilbert geometries on uniformly convex domains with $\boldsymbol{C}^{\mathbf{2}}$-boundary. Let $\mathbb{E}^{n}=\left(\mathbb{R}^{n}, d_{e}\right)=\left(\mathbb{R}^{n},|\cdot|\right)$ denote the $n$-dimensional Euclidean space. For the Euclidean distance of $x, y \in \mathbb{E}^{n}$ we write $|x y|$, and for the line segment between $x$ and $y$ we write $[x, y]$, while $L(x, y)$ denotes the whole straight line in $\mathbb{E}^{n}$ through $x$ and $y$.

Given an open bounded convex domain $D \subset \mathbb{E}^{n}$ with boundary $\partial D \subset \mathbb{E}^{n}$ and some $\kappa<0$ the Hilbert metric $h_{\kappa}^{D}: D \times D \longrightarrow \mathbb{R}_{0}^{+}$is defined as follows. For $x, y \in D$ one defines

$$
h_{\kappa}(x, y):=h_{\kappa}^{D}(x, y):=\left\{\begin{array}{cc}
\frac{1}{\sqrt{-\kappa}} \log \frac{\left|y \xi_{x, y}\right|\left|x \xi_{y, x}\right|}{\left|x \xi_{x, y}\right|\left|y \xi_{y, x}\right|} & \text { if } x \neq y, \\
0 & \text { if } x=y
\end{array}\right.
$$

where $\xi_{x, y} \in L(x, y) \cap \partial D$ is uniquely determined by the condition $\left|\xi_{x, y} x\right|<\left|\xi_{x, y} y\right|$ $\left(\xi_{y, x} \in L(x, y) \cap \partial D\right.$ by $\left|\xi_{y, x} x\right|>\left|\xi_{y, x} y\right|$, respectively). The expression

$$
\frac{\left|y \xi_{x, y}\right|\left|x \xi_{y, x}\right|}{\left|x \xi_{x, y}\right|\left|y \xi_{y, x}\right|}
$$

is called the cross ratio of the four collinear ordered points $\xi_{x, y}, x, y, \xi_{y, x}$ and is invariant under projective transformations. For the basic properties of the distance 
$h_{\kappa}$ see [Busemann 1955; de la Harpe 1993]; for example, the topology induced by $h_{\kappa}$ on $D$ coincides with the subspace topology inherited from $\mathbb{E}^{n}$. We shall refer to the metric space $\left(D, h_{\kappa}\right)$ as a Hilbert geometry.

Note that if $D$ is a ball or an ellipsoid, the associated Hilbert metric space $\left(D, h_{\kappa}\right)$ is isometric to the real hyperbolic space of constant sectional curvature $\kappa$ of corresponding dimension.

Now let $D \subset \mathbb{R}^{n}$ be an open bounded convex domain with boundary of class $C^{2}$. Let further $\rho: \mathbb{R}^{n} \longrightarrow \mathbb{R}$ be a $C^{2}$-function satisfying $\left.\rho\right|_{D}>0,\left.\rho\right|_{\partial D}=0$, and $\left.\rho\right|_{\mathbb{R}^{n} \backslash D}<0$ such that its gradient $\nabla \rho$ is a unit vector field normal to $\partial D$ and directed inside $D$. By $W_{x}: T_{x} \partial D \longrightarrow T_{x} \partial D$ we denote the curvature (or Weingarten) operator which assigns to each $v \in T_{x} \partial D$ the directional derivative of $\nabla \rho$ in direction $v$. From this curvature operator one obtains the second fundamental form $I I_{x}$ as the following bilinear form on $T_{x} \partial D$ :

$$
I I_{x}(v, w)=\left\langle w, W_{x}(v)\right\rangle=\sum_{i, j=1}^{n} \frac{\partial^{2} \rho}{\partial x^{i} \partial x^{j}} v_{i} w_{j} \quad \text { for } v, w \in T_{x} \partial D .
$$

We call $k_{x}(u):=I I_{x}(u, u)$ the normal curvature of $\partial D$ at $x$ in the direction of the unit tangent vector $u$.

In the case where the curvature of $\partial D$ is nonzero everywhere, that is, where $I I$ is positive definite everywhere, there exists some constant $k_{D}>0$ such that

$$
k_{D}^{-1} \leq k_{x}\left(\frac{u}{\|u\|}\right) \leq k_{D} \quad \text { for } x \in \partial D, u \in T_{x} \partial D .
$$

2.2. Gromov hyperbolic spaces and their boundaries at infinity. For $X$ a metric space, the Gromov product of two points of $X$ with respect to a third is defined by

$$
(x \cdot y)_{o}:=\frac{1}{2}(|x o|+|y o|-|x y|) \text { for } o, x, y \in X .
$$

The space $X$ is called Gromov hyperbolic if there exists $\delta \geq 0$ such that

$$
(x \cdot y)_{o} \geq \min \left\{(x \cdot z)_{o},(z \cdot y)_{o}\right\}-\delta \text { for } o, x, y, z \in X .
$$

This notion of Gromov hyperbolicity is a rough-isometry invariant, and the objects of interest in this asymptotic theory are the corresponding rough-isometry classes rather than the spaces themselves.

To a Gromov hyperbolic metric space one associates a boundary at infinity, endowed with a certain quasimetric. For a broad class of Gromov hyperbolic spaces (those satisfying the visuality assumption - see below), the bi-Lipschitz class of this quasimetric canonically corresponds to the rough isometry class of the space.

Now let $X$ be a Gromov hyperbolic metric space. A sequence $\left\{x_{i}\right\}$ of points $x_{i} \in X$ converges to infinity if $\lim _{i, j \rightarrow \infty}\left(x_{i} \cdot x_{j}\right)_{o}=\infty$. Two sequences $\left\{x_{i}\right\},\left\{x_{i}^{\prime}\right\}$ 
that converge to infinity are considered equivalent if $\lim _{i}\left(x_{i} \cdot x_{i}^{\prime}\right)_{o}=\infty$. Using the $\delta$ inequality (2), one easily sees that this defines an equivalence relation for sequences in $X$ converging to infinity. The boundary at infinity $\partial_{\infty} X$ of $X$ is defined as the set of equivalence classes of sequences converging to infinity.

For points $\xi, \xi^{\prime} \in \partial_{\infty} X$ one defines their Gromov product with respect to the basepoint $o \in X$ by

$$
\left(\xi \cdot \xi^{\prime}\right)_{o}:=\inf \liminf _{i \rightarrow \infty}\left(x_{i} \cdot x_{i}^{\prime}\right)_{o}
$$

where the infimum is taken over all sequences $\left\{x_{i}\right\} \in \xi$ and $\left\{x_{i}^{\prime}\right\} \in \xi^{\prime}$.

It is a well-known fact (see for instance the remark following [Bridson and Haefliger 1999, Definition 1.19]) that in the geodesic setting the Gromov product $\left(\xi \cdot \xi^{\prime}\right)_{o}$ roughly measures the distance of $o$ to the geodesic connecting $\xi$ to $\xi^{\prime}$. As we are going to use this fact later on, we formulate it as follows:

Lemma 2.1. Fix $\delta>0$. Then there exists a constant $K$ such that if $(X, d)$ is a proper geodesic Gromov hyperbolic space satisfying the $\delta$-inequality (2), then $\left|d\left(x, \operatorname{im}\{\gamma\}-\left(\xi \cdot \xi^{\prime}\right)_{x}\right)\right|<k$ for all $x \in X, \xi, \xi^{\prime} \in \partial_{\infty} X$ and every geodesic line $\gamma$ in $(X, d)$ with $c(-\infty)=\xi$ and $c(\infty)=\xi^{\prime}$.

From the inequality (2) it immediately follows that $\rho_{o}: \partial_{\infty} X \times \partial_{\infty} X \longrightarrow \mathbb{R}_{0}^{+}$, given by $\rho_{o}\left(\xi, \xi^{\prime}\right):=e^{-\left(\xi \cdot \xi^{\prime}\right)_{o}}$, is a $e^{\delta}$-quasimetric, that is,

$$
\rho_{o}\left(\xi, \xi^{\prime}\right) \leq e^{\delta} \max \left\{\rho_{o}\left(\xi^{\prime}, \xi^{\prime \prime}\right), \rho_{o}\left(\xi^{\prime \prime}, \xi^{\prime}\right)\right\} \quad \text { for } \xi, \xi^{\prime}, \xi^{\prime \prime} \in \partial_{\infty} X .
$$

It is directly clear from the definition of the boundary quasimetrics that Gromov hyperbolic spaces $X$ and $X^{\prime}$ which are rough-isometric to each other,

$$
X \stackrel{\text { rough }}{\cong} X^{\prime},
$$

give rise to boundary quasimetric spaces $\left(\partial_{\infty} X, \rho_{o}\right)$ and $\left(\partial_{\infty} X^{\prime}, \rho_{o^{\prime}}\right)$ which are bi-Lipschitz equivalent,

$$
\left(\partial_{\infty} X, \rho_{o}\right) \stackrel{\text { bi-Lip }}{\cong}\left(\partial_{\infty} X^{\prime}, \rho_{o^{\prime}}\right)
$$

For the converse statement to be true, it is clear that one has to ask the boundary somehow to represent the entire space. More precisely, recall that a metric space is called roughly geodesic if there exists some $k \geq 0$ such that any two points in the space can be joined by a $k$-rough geodesic, that is, a $k$-rough isometric embedding of a closed interval. A Gromov hyperbolic space $X$ is called visual if for some $o \in X$ and some $k \geq 0$ every point $x \in X$ lies on a $k$-rough geodesic ray initiating in $o$. In particular, a visual Gromov hyperbolic space is roughly geodesic.

Bonk and Schramm [2000] described the morphism classes of the spaces on the one hand, and those of their boundaries, on the other hand, which correspond to 
each other under the assumption of visuality. The statement we will refer to can also be deduced as a corollary of [Buyalo and Schroeder 2007, Theorem 7.1.2].

Theorem 2.2 [Bonk and Schramm 2000; Buyalo and Schroeder 2007, Theorem 7.1.2]. Let $X$ and $X^{\prime}$ be visual Gromov hyperbolic spaces, and let $o \in X$ as well as $o^{\prime} \in X^{\prime}$. Then

$$
X \stackrel{\text { rough }}{\cong} X^{\prime} \Longleftrightarrow\left(\partial_{\infty} X, \rho_{o}\right) \stackrel{\text { bi-Lip }}{\cong}\left(\partial_{\infty} X^{\prime}, \rho_{o^{\prime}}\right) .
$$

Note that in the case where the Gromov hyperbolic metric space is a CAT $(-1)$ space, the quasimetric $\rho_{o}$ indeed satisfies the triangle inequality and hence is a metric. This was shown by Bourdon [1995]. In particular, consider the real hyperbolic space $\mathbb{U}^{n}$ in the Poincaré ball model. Then the Bourdon metric $\rho_{o}$ with respect to the center of the ball $o$ is precisely given by half the Euclidean metric on $\partial_{\infty} \mathbb{H}^{n}=S^{n-1} \subset \mathbb{E}^{n}$ [Buyalo and Schroeder 2007, p. 21].

Finally note that for a Gromov hyperbolic Hilbert geometry $\left(D, h_{D}\right)$, the Gromov boundary can naturally be identified with $\partial D$, which follows from [Karlsson and Noskov 2002, Theorem 5.2] and [Foertsch and Karlsson 2005, Proposition 2].

Moreover, Hilbert geometries are visual. In fact, for any basepoint $o \in D$, every $x \in D$ lies on a geodesic ray initiating in $o$.

\section{Four elementary geometric lemmata}

This section contains the proofs of four elementary geometric lemmata, which will be referred to in the proof of Theorem 1.1 in Section 4. The complete section may be skipped at a first reading. The statements are not surprising, but we provide the proofs for the convenience of the reader.

Lemma 3.1. Let $\gamma:[0, a] \longrightarrow \mathbb{E}^{2}$ be an arc-length parameterized straight line segment of length $0<a \leq 2 \rho$ in a ball $B(r, \rho)$ around the origin $o \in \mathbb{E}^{2}$ with $\gamma(0), \gamma(a) \in \partial B(o, \rho)$, and denote by $l=l(\rho, a)>0$ the distance of $\gamma(a / 2)$ to the two-point set $L(o, \gamma(a / 2)) \cap \partial B(o, \rho)$, for $a<2 \rho$, and $l=\rho$ otherwise. Then

$$
\frac{1}{\Lambda(\rho)} \sqrt{l(\rho, a)} \leq a \leq \Lambda(\rho) \sqrt{l(\rho, a)} \text { for } a \in[0,2 \rho],
$$

with $\Lambda(\rho):=\max \{2 \sqrt{2 \rho}, 1 /(2 \sqrt{\rho})\}$.

Proof. This immediately follows from $a=2 \sqrt{2 \rho-l(\rho, a)} \sqrt{l(\rho, a)}$ and $0 \leq$ $l(\rho, a) \leq \rho$.

Now let $R>r>0$ and let $S$ be a straight line segment in $\mathbb{E}^{2}$ of length $x$, the endpoints of which lie on $\partial B(o, R) . \overline{B(o, R)} \backslash S$ consists of two connected components $\tilde{B}$ and $\hat{B}$. For $x<r$, let $\tilde{B}(S)$ be the component disjoint from $B(o, R-r)$. Given $p \in B(o, R-r)$ and $q \in S$, define

$$
w=w(p, q):=L(p, q) \cap \tilde{B}(S) \cap \partial B(o, R)
$$




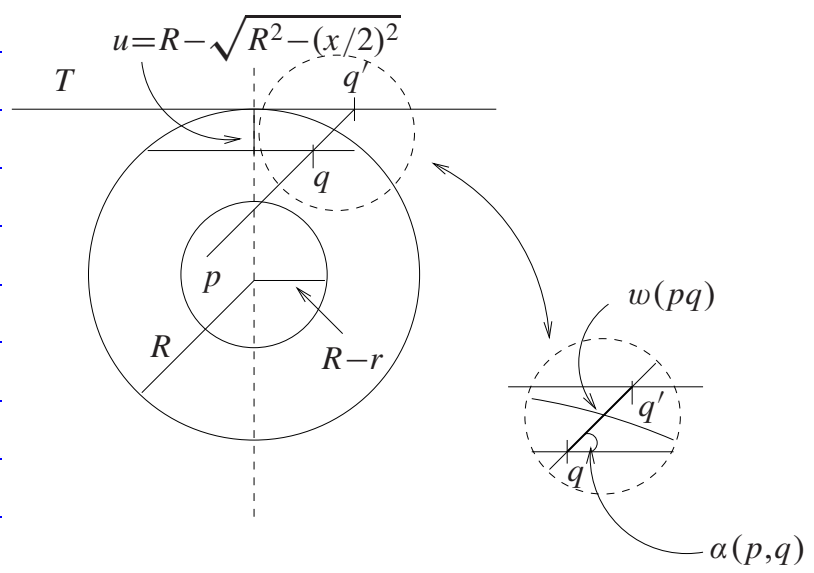

Figure 1. Notation in Lemma 3.2.

and set

$$
m=m(x, R, r):=\max _{\substack{p \in B(o, R-r) \\ q \in S}}|q w(p, q)| .
$$

Lemma 3.2. Fix $R>r>0$. Then

$$
m(x, R, r) \leq \tilde{\Lambda}\left(R-\sqrt{R^{2}-(x / 2)^{2}}\right)
$$

for $\tilde{\Lambda}=\tilde{\Lambda}(r, R):=\sin ^{-1}(\arctan r /(4 R))$.

Proof. For $p \in B(o, R-r)$ and $q \in S$, let $\alpha=\alpha(p, q)$ denote the angle $\alpha(p, q):=$ $\left\llcorner_{q}(L(p, q), S) \in(0, \pi / 2]\right.$. Further, let $T$ denote the tangential line to $\partial \tilde{B}(S) \backslash S$ parallel to $S$, and set $q^{\prime}:=T \cap L(p, q)$ and $v:=\left|q q^{\prime}\right|$. Then

$$
|q w(p, q)|<v=\frac{u}{\sin \alpha(p, q)} \quad \text { with } \quad u:=R-\sqrt{R^{2}-(x / 2)^{2}} .
$$

Therefore it remains to prove that there exists $\alpha_{0}>0$ such that $\alpha(p, q) \geq \alpha_{0}$ for all $p \in B(o, R-r)$ and $q \in S$.

Since $x<r$, we deduce $u<r / 2$ and therefore $\operatorname{dist}(S, \partial B(o, R-r))>r / 2$. It follows that we can choose

$$
\alpha_{0}:=\arctan \frac{r / 2}{2 R}=\arctan \frac{r}{4 R} .
$$

Let $\rho_{2}>\rho_{1}>0$ be fixed and $C_{\rho_{2}}, C_{\rho_{1}}$ be circles in $\mathbb{E}^{2}$ of radius $\rho_{2}$ and $\rho_{1}$, respectively, such that $\#\left(C_{\rho_{1}} \cap C_{\rho_{2}}\right)=1$ with the center $o_{\rho_{1}}$ of $C_{\rho_{1}}$ in the bounded component of $\mathbb{R}^{2} \backslash C_{2}$. Let $q:=C_{\rho_{1}} \cap C_{\rho_{2}}$, and denote by $o_{\rho_{2}}$ the center of $C_{\rho_{2}}$. Further, let $L_{0}$ be the straight line through $o_{\rho_{2}}$ orthogonal to $L\left(q, o_{\rho_{2}}\right)$. By $H$ we denote the half-space in $\mathbb{E}^{2}$ defined by $L_{0}$ such that $H$ contains the center $o_{\rho_{1}}$ of $C_{\rho_{1}}$. Now let $L_{t} \subset H$ be the parallel to $L_{0}$ in distance $t$ of $o_{\rho_{2}}$ for all $t \in\left[0, \rho_{2}\right)$ 


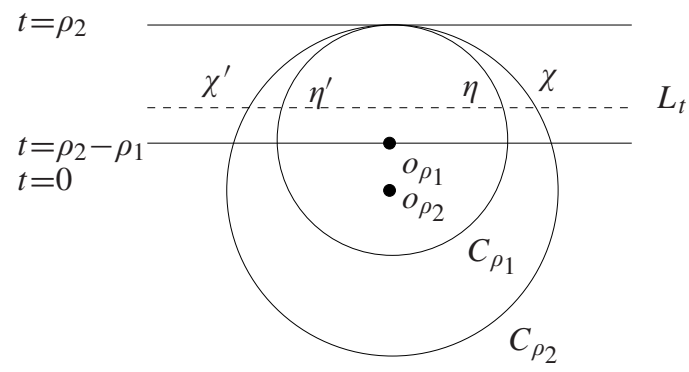

Figure 2. Illustration of the situation considered in Lemma 3.3.

and define $\chi_{t}, \chi_{t}^{\prime}, \eta_{t}, \eta_{t}^{\prime} \in \mathbb{E}^{2}$ via $\left\{\chi_{t}, \chi_{t}^{\prime}\right\}=L_{t} \cap C_{\rho_{2}}$ and $\left\{\eta_{t}, \eta_{t}^{\prime}\right\}=L_{t} \cap C_{\rho_{1}}$ for all $t \in\left[\rho_{2}-\rho_{1}, \rho_{2}\right)$.

Lemma 3.3. Let $\rho_{2}>\rho_{1}>0$. Then $\left|\chi_{t} \chi_{t}^{\prime}\right| \leq \hat{\Lambda}\left|\eta_{t} \eta_{t}^{\prime}\right|$ for all $t \in\left[\rho_{2}-\rho_{1}, \rho_{2}\right)$, with $\hat{\Lambda}=\hat{\Lambda}\left(\rho_{1}, \rho_{2}\right):=\sqrt{\left(2 \rho_{2}-\rho_{1}\right) / \rho_{1}}$.

Proof. Consider the function $f:\left[\rho_{2}-\rho_{1}, \rho_{2}\right) \longrightarrow \mathbb{R}^{+}$given by

$$
f(t):=\frac{\left|\chi_{t} \bar{\chi}_{t}\right|^{2}}{\left|\eta_{t} \bar{\eta}_{t}\right|^{2}}=\frac{\rho_{2}^{2}-t^{2}}{\rho_{1}^{2}-\left(t-\left(\rho_{2}-\rho_{1}\right)\right)^{2}} \quad \text { for all } \quad t \in\left[\rho_{2}-\rho_{1}, \rho_{2}\right) .
$$

With $f^{\prime}(t) \neq 0$ for all $t \in\left(\rho_{2}-\rho_{1}, \rho_{2}\right)$, as well as

$$
\lim _{t \rightarrow \rho_{2}} f(t)=\rho_{2} / \rho_{1} \leq\left(2 \rho_{2}-\rho_{1}\right) / \rho_{1}=f\left(\rho_{2}-\rho_{1}\right),
$$

the claim follows.

Lemma 3.4. Let $D$ be a bounded, convex domain in $\mathbb{E}^{n+1}$ with $C^{1}$-boundary $\partial D$. Then $\left(\partial D,\left.d_{e}\right|_{\partial D \times \partial D}\right)$ is bi-Lipschitz equivalent to $\left(S^{n}, d_{e} \mid S^{n}\right)$.

Proof. Let $x \in D$ and let $r>0$ be such that $B_{r}(x) \subset D$. Consider the map $\varphi:\left(\partial D,\left.d_{e}\right|_{\partial D \times \partial D}\right) \longrightarrow\left(\partial B_{r}(x),\left.d_{e}\right|_{\partial B_{r}(x) \times \partial B_{r}(x)}\right)$, given by

$$
\xi \mapsto \eta \in L(x, \xi) \cap \partial B_{r}(x) \text { with }|\eta \xi|=\operatorname{dist}\left(\xi, L(x, \xi) \cap \partial B_{r}(x)\right) .
$$

Obviously, $\left|\xi \xi^{\prime}\right| \leq\left|\varphi(\xi) \varphi\left(\xi^{\prime}\right)\right|$ for all $\xi, \xi^{\prime} \in \partial D$. Moreover, for all $\alpha>0$ there exists $\mu(\alpha)$ such that

$$
\left|\xi \xi^{\prime}\right| \geq \mu(\alpha)\left|\varphi(\xi) \varphi\left(\xi^{\prime}\right)\right| \text { for } \xi, \xi^{\prime} \in \partial D, \text { with } \quad L_{x}\left(\xi, \xi^{\prime}\right) \geq \alpha .
$$

Therefore we only have to consider angles approaching zero.

Let $R_{\xi, x}:=|\xi x|$ and let $R_{x}:=\left\{\max R_{\xi x} \mid \xi \in \partial D\right\}$. Let further $T_{\xi}$ denote the tangent to $\partial D$ at $\xi \in \partial D$ and set $\gamma_{x \xi}:=L_{\xi}\left(T_{\xi}, L(x, \xi)\right) \in\left(0, \frac{\pi}{2}\right)$. Then, since $D$ is $C^{1}$ and convex and $\partial D$ is compact, there exists $\gamma_{0}>0$ such that

$$
\inf \left\{\gamma_{x \xi} \mid \xi \in \partial D\right\}=\min \left\{\gamma_{x \xi} \mid \xi \in \partial D\right\} \geq \gamma_{0} .
$$



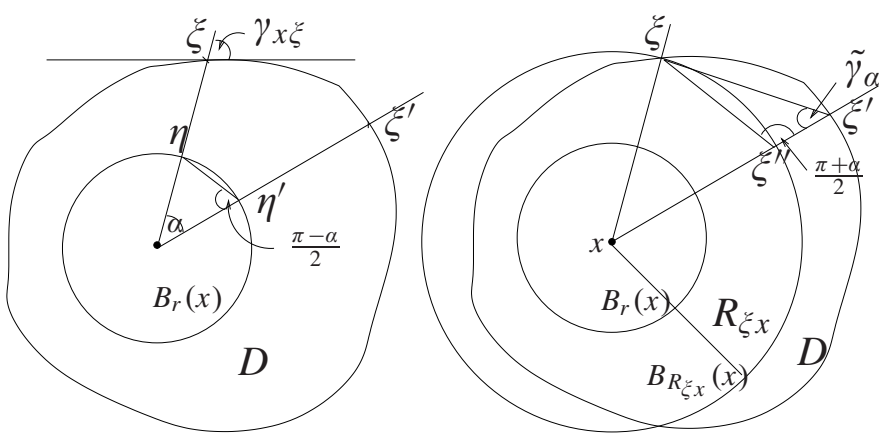

Figure 3. Notation used in the proof of Lemma 3.4.

Now consider $\xi, \xi^{\prime} \in \partial D$ with $L_{x}\left(\xi, \xi^{\prime}\right)=\alpha$. Let $C_{x, \xi, \xi^{\prime}}\left(R_{\xi, x}\right)$ be the circle in $\operatorname{span}\left\{x, \xi, \xi^{\prime}\right\}$ of radius $R_{\xi, x}$ and center $x$, and let $\xi^{\prime \prime}:=L\left(x, \xi^{\prime}\right) \cap C_{x, \xi, \xi^{\prime}}\left(R_{\xi, x}\right)$ with $\left|\xi^{\prime \prime} x\right|-\left|\xi^{\prime} x\right||=| \xi^{\prime} \xi^{\prime \prime} \mid$. Since $L_{\xi}\left(x, \xi^{\prime \prime}\right)=\frac{1}{2}(\pi-\alpha)=L_{\xi^{\prime \prime}}(x, \xi)$, we find

$$
\frac{L_{\alpha}^{\xi}}{\sin \frac{1}{2}(\pi-\alpha)}=\frac{l_{\alpha}^{\xi}}{\sin \tilde{\gamma}_{\alpha}},
$$

where $L_{\alpha}^{\xi}:=\left|\xi^{\prime} \xi\right|, l_{\alpha}^{\xi}:=\left|\xi^{\prime \prime} \xi\right|$ and $\tilde{\gamma}_{\alpha}:=L_{\xi^{\prime}}\left(\xi^{\prime \prime}, \xi\right)$.

Now, since $\sin \tilde{\gamma}_{\alpha} \rightarrow \sin \gamma_{x, \xi} \geq \sin \gamma_{0}$ as $\alpha \rightarrow 0$, it follows that for all $\xi \in \partial D$ there exists $\alpha_{0}(\xi)$ such that

$$
L_{\alpha}^{\xi} \leq \frac{\sin \frac{1}{2}(\pi-\alpha)}{\sin \frac{1}{2} \gamma_{0}} l_{\alpha}^{\xi}
$$

for all $\alpha \leq \alpha_{0}(\xi)$. Thus, since $\partial D$ is compact, there also exist $\alpha_{0}>0$ as well as $\mu>0$ such that $L_{\alpha}^{\xi} \leq \mu l_{\alpha}^{\xi}$ for all $\alpha<\alpha_{0}$, from which the claim follows.

\section{Proof of Theorem 1.1}

We prove that $\left(D, h_{-1}\right) \stackrel{\text { rough }}{\cong} \mathbb{T}_{-1}^{n}$. The rest of the claim follows as usual by merely rescaling the metric.

From [Karlsson and Noskov 2002, Theorem 5.2] and [Foertsch and Karlsson 2005, Proposition 2] it follows that the Gromov boundary at infinity of $\left(D, h_{-1}\right)$ can naturally be identified with $\partial D \subset \mathbb{D}^{n}$. The main goal of this proof is to verify that for $x \in D$ the visual quasimetric $\rho_{x}$ on $\partial D$ is bi-Lipschitz equivalent to the restriction of the Euclidean metric $d_{e}=|\cdot|$ to $\partial D$.

Let $k_{D}$ be as in (1) and set $\rho_{1}:=\sqrt{k_{D}^{-1}}$ and $\rho_{2}:=\sqrt{k_{D}}$. Fix $x \in D$ and let $R_{x}>r_{x}>0$ be such that $B\left(x, r_{x}\right) \subset D \subset B\left(x, R_{x}\right)$. We want to show that 


$$
\left.\rho_{x} \stackrel{\text { bi-Lip }}{\cong} d_{e}\right|_{\partial D}=:\left.|\cdot|\right|_{\partial D}
$$

(i) In the first step we establish that

$$
\text { there exists } \lambda>0 \text { such that } e^{-\left(\xi \cdot \xi^{\prime}\right)_{x}} \geq \frac{1}{\lambda}\left|\xi \xi^{\prime}\right|, \quad \text { for all } \xi, \xi^{\prime} \in \partial D \text {. }
$$

Let therefore $\xi, \xi^{\prime} \in \partial D$ and $y \in\left[\xi, \xi^{\prime}\right]$ satisfying $d(x, y)=\operatorname{dist}\left(x,\left[\xi, \xi^{\prime}\right]\right)$. Note that for $x \in\left[\xi, \xi^{\prime}\right]$ we have $e^{-\left(\xi^{\prime} \cdot \xi^{\prime}\right)_{x}}=1$ and $e^{-\left(\xi \cdot \xi^{\prime}\right)_{x}} \geq \frac{1}{\lambda}\left|\xi \xi^{\prime}\right|$ holds for $\lambda \geq \operatorname{diam} D$. Therefore we can assume in the following without loss of generality that $x \notin\left[\xi, \xi^{\prime}\right]$.

Now let $y^{\prime} \in\left[\xi, \xi^{\prime}\right] \cap D$ be arbitrary and $A, B \in L\left(x, y^{\prime}\right) \cap \partial D$ be defined via $|A x|<|A y|$ and $|B y|<|B x|$. Then, due to Lemma 2.1 and the inequalities $r_{x} \leq|x A|,\left|y^{\prime} A\right|,|x B| \leq 2 R_{x}$, we deduce the existence of $\tilde{\lambda}_{1}, \tilde{\lambda}_{2}>0$ only depending on $\left(D, h_{-1}\right), r_{x}$ and $R_{x}$ such that

$$
e^{-(\xi \cdot \xi)_{x}} \geq \frac{1}{\tilde{\lambda}_{1}} e^{-h_{1}(x, y)} \geq \frac{1}{\tilde{\lambda}_{1}} e^{-h_{1}\left(x, y^{\prime}\right)}=\frac{1}{\tilde{\lambda}_{1}} \sqrt{\frac{|x A|\left|y^{\prime} B\right|}{|x B|\left|y^{\prime} A\right|}} \geq \frac{1}{\tilde{\lambda}_{2}} \sqrt{\left|y^{\prime} B\right|} .
$$

Thus it remains to show that there exists $\tilde{\lambda}_{3}>0$ only depending on $\left(D, h_{-1}\right), r_{x}$ and $R_{x}$ such that for all $\xi, \xi^{\prime} \in \partial D$ there exists $y^{\prime}$ as above satisfying

$$
\sqrt{\left|y^{\prime} B\right|} \geq \frac{1}{\tilde{\lambda}_{3}}\left|\xi \xi^{\prime}\right|
$$

To prove this, consider the two-dimensional plane $\Sigma$ spanned by $x, \xi, \xi^{\prime}$. The set $(\Sigma \cap D) \backslash\left[\xi, \xi^{\prime}\right]$ consists of two connected components. Denote by $\tilde{\Sigma}$ the connected component of this set not containing $x$. Since $\partial D$ is $C^{2}$, there exists $B \in \partial \tilde{\Sigma} \backslash\left[\xi, \xi^{\prime}\right] \subset \partial D$ such that the tangent $T(B)$ of $\partial \tilde{\Sigma}$ at $B$ is parallel to $\left[\xi, \xi^{\prime}\right]$.

Let $T(B)^{\perp} \subset \Sigma$ denote the straight line through $B$ orthogonal to $T(B)$. Let further $C_{\rho_{2}}$ be the circle of radius $\rho_{2}$ in $\Sigma$ through $B$, tangent to $T(B)$, which

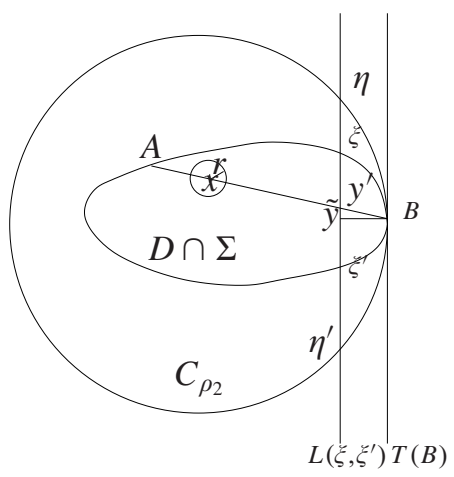

Figure 4. Situation in step (i) of the proof. 
lies on the same side of $T(B)$ in $\Sigma$ as $D$ does. Now set $y^{\prime}:=[x, B] \cap\left[\xi, \xi^{\prime}\right]$, $\tilde{y}:=T(B)^{\perp} \cap\left[\xi, \xi^{\prime}\right]$ as well as $\eta, \eta^{\prime} \in L\left(\xi, \xi^{\prime}\right) \cap C_{\rho_{2}}$ such that $|\eta \xi|<\left|\eta \xi^{\prime}\right|$ and $\left|\eta^{\prime} \xi^{\prime}\right|<\left|\eta^{\prime} \xi\right|$.

Now we consider two cases:

- If $\operatorname{dist}\left(\left[\xi, \xi^{\prime}\right], T(B)\right) \geq \rho_{2}$, then (3) holds trivially for $\left|y^{\prime} B\right|$ as above once $\lambda_{3} \geq \operatorname{diam}(D) / \sqrt{\rho_{2}}$.

- If $\operatorname{dist}\left(\left[\xi, \xi^{\prime}\right], T(B)\right)<\rho_{2}$ we find with Lemma 3.1:

$$
\left|\xi \xi^{\prime}\right| \leq\left|\eta \eta^{\prime}\right| \leq \Lambda\left(\rho_{2}\right) \sqrt{l\left(\rho_{2},\left|\eta \eta^{\prime}\right|\right)}=\Lambda\left(\rho_{2}\right) \sqrt{|\tilde{y} B|} \leq \Lambda\left(\rho_{2}\right) \sqrt{\left|y^{\prime} B\right|} .
$$

(ii) In the second step we establish that

$$
\text { there exists } \lambda>0 \text { such that } e^{-\left(\xi^{\prime} \xi^{\prime}\right)_{x}} \leq \lambda\left|\xi \xi^{\prime}\right|, \quad \text { for all } \xi, \xi^{\prime} \in \partial D \text {. }
$$

To do this, we choose $x$ to be particularly nice: Let $E \in \partial D$, take the ball $B_{\rho_{1}}$ of radius $\rho_{1}$ tangent to the tangent hyperplane $H(E)$ of $\partial D$ at $E$ such that $B_{\rho_{1}}{ }^{\circ} \subset D$, and let $x$ be the center of $B_{\rho_{1}}$. With $x$ defined like this we have $|x \xi| \geq \rho_{1}$ for all $\xi \in \partial D$.

Now, for $\xi, \xi^{\prime} \in \partial D, \xi \neq \xi^{\prime}$, arbitrarily choose $y$ as above and let $\bar{x}=\xi_{x, y}, \bar{y}=$ $\xi_{y, x} \in \partial D$ be as in the definition of the Hilbert distance between $x$ and $y$. Once again we can assume without loss of generality that $x \notin\left[\xi, \xi^{\prime}\right]$. Due to Lemma 2.1 and $r_{x} \leq|x \bar{x}|,|y \bar{x}|,|x \bar{y}| \leq 2 R_{x}$ we deduce the existence of $\tilde{\lambda}_{4}, \tilde{\lambda}_{5}>0$ only depending on $\left(D, h_{-1}\right), r_{x}$ and $R_{x}$ such that

$$
e^{-\left(\xi \cdot \xi^{\prime}\right)_{x}} \leq \tilde{\lambda}_{4} e^{-h_{1}(x, y)}=\tilde{\lambda}_{4} \sqrt{\frac{|x \bar{x}| \cdot|y \bar{y}|}{|x \bar{y}| \cdot|y \bar{x}|}} \leq \tilde{\lambda}_{5} \sqrt{|y \bar{y}|} .
$$

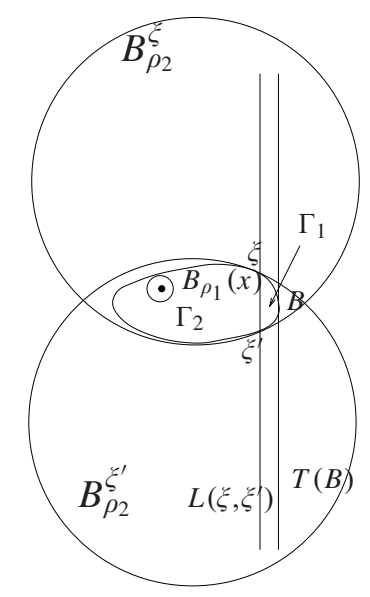

Figure 5. Notation in the proof of step (ii), with $i_{0}=1$. 
Thus it remains to show that there exists $\tilde{\lambda}_{6}>0$ only depending on $\left(D, h_{1}\right), r_{x}$ and $R_{x}$ such that for all $\xi, \xi^{\prime} \in \partial D$, the inequality $\sqrt{|y \bar{y}|} \leq \tilde{\lambda}_{6}\left|\xi \xi^{\prime}\right|$ holds.

Since $|y \bar{y}| \leq \operatorname{diam}(D)$, it suffices to restrict our attention to those $\xi$, $\xi^{\prime} \in \partial D$ satisfying $\left|\xi \xi^{\prime}\right|<1 / n$ for arbitrary but fixed $n \in \mathbb{N}$. We choose $n$ as follows.

Let $\xi, \xi^{\prime} \in \partial D$ and $\Sigma:=\operatorname{span}\left\{x, \xi, \xi^{\prime}\right\}$ as above. Let further $B_{\rho_{2}}^{\xi}$ and $B_{\rho_{2}}^{\xi^{\prime}}$ denote the balls of radius $\rho_{2}$ through $\xi$ and $\xi^{\prime}$ in $\Sigma$ tangential to the tangents of $\partial D \cap \Sigma$ in $\xi$ and $\xi^{\prime}$, respectively, such that $D \subset B_{\rho_{2}}^{\xi} \cap B_{\rho_{2}}^{\xi}=: \sigma$.

Then $\partial \sigma \backslash\left\{\xi, \xi^{\prime}\right\}$ consists of two arcs $\gamma_{1}$ and $\gamma_{2}$ of length $l\left(\gamma_{1}\right)$ and $l\left(\gamma_{2}\right)$, respectively. Since $\rho_{1}$ and $\rho_{2}$ are fixed, it is immediate that there exists an $n_{0}=n_{0}\left(\rho_{1}, \rho_{2}\right)$ such that from $\left|\xi \xi^{\prime}\right|<\frac{1}{n_{0}}$, it follows that $\min \left\{l\left(\gamma_{1}\right), l\left(\gamma_{2}\right)\right\}<\rho_{1}$. Let us now assume without loss of generality (see above) that $\left|\xi \xi^{\prime}\right|<\frac{1}{n_{0}}$.

We take $i_{0} \in\{1,2\}$ such that $l\left(\gamma_{i_{0}}\right)=\min \left\{l\left(\gamma_{1}\right), l\left(\gamma_{2}\right)\right\}<\rho_{1}$ and denote the connected components of $\sigma \backslash\left\{\xi, \xi^{\prime}\right\}$ by $\Gamma_{1}$ and $\Gamma_{2}$ such that $\partial \Gamma_{i}=\left[\xi, \xi^{\prime}\right] \cup \gamma_{i}$, $i=1,2$.

Since for each point $z \in \Gamma_{i_{0}}$ we have $\operatorname{dist}\{z, \partial D\}<\rho_{1}$, we deduce $x \notin \Gamma_{i_{0}}$ and thus $\bar{y} \in \Gamma_{i_{0}}$ for $\bar{y}=\xi_{y, x}$, as in the definition of the Hilbert distance between $x$ and $y$.

Now let $B \in \Gamma_{i_{0}}$ and $T(B)$ be as in (i), and denote by $B_{\rho_{1}}$ and $B_{\rho_{2}}$ the balls in $\Sigma$ of radii $\rho_{1}$ and $\rho_{2}$ through $B$, tangent to $T(B)$, which lie on the same side of $T(B)$ in $\Sigma$ as $D$ does. We denote the center of $B_{\rho_{1}}$ by $o_{\rho_{1}}$ and write $T_{\rho_{1}}$ for the straight line through $o_{\rho_{1}}$ parallel to $T(B)$. Further, let $S$ be the strip bounded by $T(B)$ and $T_{\rho_{1}}$. Since $B \in \Gamma_{i_{0}}$ and thus $|\xi B|,\left|\xi^{\prime} B\right|<\rho_{1}$, it follows that $\xi, \xi^{\prime} \in\left(S \cap B_{\rho_{2}}\right) \backslash B_{\rho_{1}}^{\circ}$.

Thus we are exactly in the situation to apply Lemmata 3.1, 3.2 and 3.3. Let therefore $y^{\prime}:=T(B)^{\perp} \cap\left[\xi, \xi^{\prime}\right]$. Then we get

$$
\begin{aligned}
& \sqrt{|y \bar{y}|} \leq \tilde{\Lambda}\left(\rho_{1}, \rho_{2}\right) \cdot \sqrt{\left|y^{\prime} B\right|} \\
& \leq \tilde{\Lambda}\left(\rho_{1}, \rho_{2}\right) \cdot \Lambda\left(\rho_{2}\right) \cdot\left|\chi \chi^{\prime}\right| \\
& \leq \tilde{\Lambda}\left(\rho_{1}, \rho_{2}\right) \cdot \Lambda\left(\rho_{2}\right) \cdot \hat{\Lambda}\left(\rho_{1}, \rho_{2}\right) \cdot\left|\eta \eta^{\prime}\right| \\
& \leq \tilde{\Lambda}\left(\rho_{1}, \rho_{2}\right) \cdot \Lambda\left(\rho_{2}\right) \cdot \hat{\Lambda}\left(\rho_{1}, \rho_{2}\right) \cdot\left|\xi \xi^{\prime}\right|=: \tilde{\lambda}_{6} \cdot\left|\xi \xi^{\prime}\right|,
\end{aligned}
$$

where $\left\{\chi, \chi^{\prime}\right\}:=L\left(\xi, \xi^{\prime}\right) \cap C_{\rho_{2}}$ and $\left\{\eta, \eta^{\prime}\right\}:=L\left(\xi, \xi^{\prime}\right) \cap C_{\rho_{1}}$ and $C_{\rho_{i}}:=\partial B_{\rho_{i}}$, $i=1,2$. Thus, applying Lemma 3.4, we have indeed established that the visual metric $\rho_{x}$ on the boundary at infinity of $\left(D, h_{-1}\right)$ is bi-Lipschitz equivalent to the angular boundary metric on $\partial \mathbb{H}_{-1}^{n}$. The claim therefore follows from Theorem 2.2 together with the obvious fact that $\left(D, h_{-1}\right)$ is visual.

\section{Acknowledgment}

It is a pleasure to thank Mario Bonk for many discussions on Gromov hyperbolic spaces in general, and particularly on the subject of Gromov hyperbolic Hilbert geometries. 


\section{References}

[Benoist 2003] Y. Benoist, "Convexes hyperboliques et fonctions quasisymétriques", Publ. Math. Inst. Hautes Études Sci. 97 (2003), 181-237. MR 2005g:53066 Zbl 1049.53027

[Benoist 2006] Y. Benoist, “Convexes hyperboliques et quasiisométries”, Geometriae Dedicata 122 (2006), 109-134. MR 2007k:20091 Zbl 1122.20020

[Bernig 2009] A. Bernig, "Hilbert geometry of polytopes", Arch. Math. (Basel) 92:4 (2009), 314324. MR 2501287 Zbl 1171.53046

[Bletz-Siebert and Foertsch 2007] O. Bletz-Siebert and T. Foertsch, "The Euclidean rank of Hilbert geometries”, Pacific J. Math. 231:2 (2007), 257-278. MR 2008j:53073 Zbl 1155.53331

[Bonk and Schramm 2000] M. Bonk and O. Schramm, "Embeddings of Gromov hyperbolic spaces", Geom. Funct. Anal. 10:2 (2000), 266-306. MR 2001g:53077 Zbl 0972.53021

[Bourdon 1995] M. Bourdon, "Structure conforme au bord et flot géodésique d'un CAT(-1)-espace", Enseign. Math. (2) 41:1-2 (1995), 63-102. MR 96f:58120 Zbl 0871.58069

[Bridson and Haefliger 1999] M. R. Bridson and A. Haefliger, Metric spaces of non-positive curvature, Grundlehren der Mathematischen Wissenschaften [Fundamental Principles of Mathematical Sciences] 319, Springer, Berlin, 1999. MR 2000k:53038 Zbl 0988.53001

[Busemann 1955] H. Busemann, The geometry of geodesics, Academic Press, New York, N. Y., 1955. MR 17,779a Zbl 0112.37002

[Buyalo and Schroeder 2007] S. Buyalo and V. Schroeder, Elements of asymptotic geometry, European Mathematical Society, Zürich, 2007. MR 2009a:53068 Zbl 1125.53036

[Colbois and Verovic 2004] B. Colbois and P. Verovic, "Hilbert geometry for strictly convex domains", Geometriae Dedicata 105 (2004), 29-42. MR 2005e:53111 Zbl 1078.52002

[Colbois and Verovic 2008] B. Colbois and P. Verovic, "Hilbert domains quasi-isometric to normed vector spaces", preprint, 2008. arXiv 0804.1619

[Colbois et al. 2008] B. Colbois, C. Vernicos, and P. Verovic, "Hilbert geometry for convex polygonal domains", preprint, 2008. arXiv 0804.1620

[Foertsch and Karlsson 2005] T. Foertsch and A. Karlsson, "Hilbert metrics and Minkowski norms", J. Geom. 83:1-2 (2005), 22-31. MR 2007e:51021 Zbl 1084.52008

[de la Harpe 1993] P. de la Harpe, "On Hilbert's metric for simplices", pp. 97-119 in Geometric group theory, Vol. 1 (Sussex, 1991), edited by G. A. Niblo and M. A. Roller, London Math. Soc. Lecture Note Ser. 181, Cambridge Univ. Press, 1993. MR 94i:52006 Zbl 0832.52002

[Karlsson and Noskov 2002] A. Karlsson and G. A. Noskov, "The Hilbert metric and Gromov hyperbolicity”, Enseign. Math. (2) 48:1-2 (2002), 73-89. MR 2003f:53061 Zbl 1046.53026

Received July 30, 2009.

THOMAS FOERTSCH

MATHEMATISCHES INSTITUT

UNIVERSITÄT BONN

53115 BONN

GERMANY

foertsch@math.uni-bonn.de

http://www.math.uni-bonn.de/people/foertsch/ 